\section{Successful resuscitation of out of hospital cardiac arrest patients in the emergency department}

SHOU-YEN CHEN • SHIH-HAO WANG • YI-MING WENG • YU-CHE CHANG • YU-JR LIN • KO-CHEN CHANG • JIUN-HAO YU
YI-MING WENG( $\bowtie)$ Department of Emergency Medicine Chang Gung Memorial Hospital and Chang Gung University College of Medicine

Department of Emergency Medicine Chang Gung Memorial Hospital No. 5 Fushing St.

Gueishan Shiang, Taoyuan, Taiwan Phone: 886-3-3281-200 ext 2505

Fax: 886-3-3287-715

E-mail:wengym33@gmail.com yiming33@adm.cgmh.org.tw

SHOU-YEN CHEN • SHIH-HAO WANG • YU-CHE CHANG • KO-CHEN CHANG • JIUN-HAO YU

Department of Emergency Medicine Chang Gung Memorial Hospital and Chang Gung University College of Medicine, Tao-Yuan, Taiwan

SHIH-HAO WANG Institute of Environmental and Occupational Health Science, National Yang-Ming University, Taipei, Taiwan

YU-JR LIN

Biostatistical Center for clinical Research Chang Gung Memorial Hospital Taoyuan, Taiwan

\begin{abstract}
Background. We examined factors associated with the successful resuscitation, in the emergency department (ED), of adult, out-of-hospital cardiac arrest (OHCA) patients.

Methods. The study cohort consisted of adult patients (over 18 years of age) who presented to the ED in 2009 with a diagnosis of cardiac arrest. Data were retrieved from the institutional database.

Results. A total of 122 adult, non-traumatic, OHCA patients were enrolled in the study. There were no significant differences between the sustained return of spontaneous circulation (ROSC) and non-sustained ROSC groups in initial body temperature $(P=0.420)$, time to successful intubation $(P=0.524)$, time to first intravenous epinephrine injection $(P=0.108)$, blood sugar levels $(P=0.122)$, hematocrit $(P=0.977)$, cardiac enzymes $(P=0.116)$ and serum sodium level $(P=0.429)$. Leukocytosis $(P=0.047)$ and cardiac rhythm of pulseless ventricular tachycardia/ ventricular fibrillation and pulseless electrical activity $(P=0.022)$, were significantly associated with sustained ROSC. In contrast, patients with more severe acidosis $(P=0.003)$ and hyperkalemia $(P<0.001)$ had a reduced likelihood of achieving sustained ROSC. After multiple variable logistic regression analysis adjusting for variables, the correlation between sustained ROSC and leukocytosis and hyperkalemia remained high (leukocytosis, $P=0.007$, odds ratio [OR] 3.655, 95\% Cl 1.422-9.395; hyperkalemia, $P=0.001$, OR 0.169, 95\% Cl 0.057-0.500)

Conclusion. Patients suffering an OHCA were appropriately resuscitated after arriving at the ED. Successful resuscitation in adult OHCA victims was determined by the patient's status, in particular their white blood cell count and potassium level.
\end{abstract}


Key words: adult out of hospital cardiac arrest, return of spontaneous circulation, in-hospital phase, hyperkalemia, leukocytosis

\section{Introduction}

\section{Background}

Out-of-hospital cardiac arrest (OHCA) poses a difficult challenge for emergency physicians. The survival rate of out-of-hospital cardiac arrest patients is low, ranging from $1.7 \%$ to $6.1 \%$. (1-4) Identification of the factors associated with sustained return of spontaneous circulation (ROSC) in adult OHCA victims may help emergency physicians to deal more effectively with such cases. Predictors of sustained ROSC in cases of adult OHCA include witness of collapse, initial cardiac rhythm, bystander cardiopulmonary resuscitation (CPR), early start of emergency medical system (EMS), early defibrillation, and a short duration of transportation. (5-9) These predictors have been studied during the pre-hospital phase and may not be available when patients arrive at the emergency department (ED). However, no previous reports have examined factors associated with ROSC in adult OHCA patients during the in-hospital phase, which may be useful as parameters for predicting the effects of resuscitation, and may aid in the further improvement of treatment methods or interventions.

Therefore, the aim of our study is to determine the factors associated with sustained ROSC in adult OHCA patients during the in-hospital phase. We hypothesized that the factors associated with sustained ROSC in adult OHCA victims, after arriving at the ED, could help physicians improve resuscitation and predict the outcome.

\section{Methods}

\section{Study Design and Setting}

This was a retrospective cohort study conducted at a university-affiliated teaching hospital with an annual ED case load of 227,000 visits. This study was approved by the Hospital Ethics Committee on Human Research. The study protocol was reviewed and

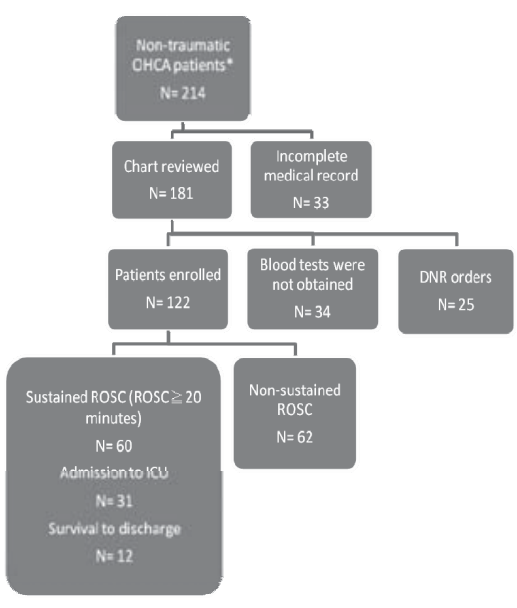

DNR, Do-not-resuscitate; ICU, Intensive care unit OHCA, out of hospital cardiac arrest; ROSC, return of spontaneous circulation.

Figure 1. Patient eligibility. Numbers of patients included and excluded from the study.

*Adults, over 18 years of age, with ICD-9CM codes for cardiac arrest 7981,7982 and 7989 , were included. Patients who were not resuscitated due to 'do-not-resuscitate (DNR)' orders and those with incomplete or lost documentation were excluded.

deemed exempt from the requirement to obtain informed consent.

Patient selection

The study cohort consisted of adult patients over 18 years of age who presented to the ED of a teaching hospital between January 1, 2009, and December 31, 2009 with the main diagnosis of cardiac arrest. Patients with International Classification of Disease, $9^{\text {th }}$ revision and Clinically Modified (ICD-9-CM) Diagnosis Code 798 Cardiac Arrest were included in the study. Patients with ICD-9CM Diagnosis Code 798.0 (Sudden Infant Death Syndrome), those who received no resuscitation due to 'do-notresuscitate (DNR)'orders, and those for whom documentation was incomplete or had been lost were excluded from the study. We reviewed 214 cases and excluded 92 patients based on the exclusion criteria; thus, 122 patients were enrolled in this study. Sustained ROSC was achieved in 60 (49.1\%) patients, and 31 (25.4\%) were admitted to the intensive care unit, with $12(9.8 \%)$ surviving to discharge. Among the 92

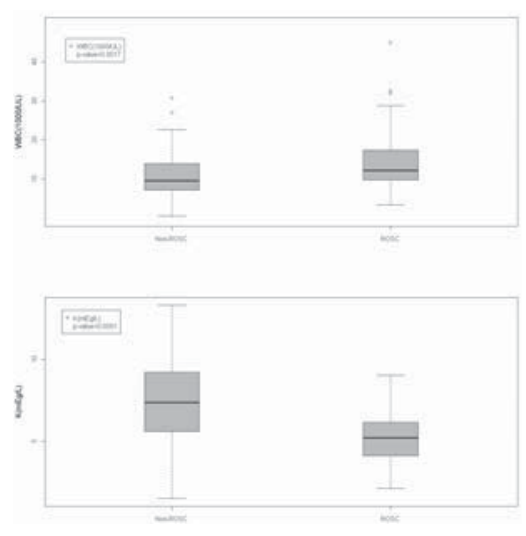

Figure 2. White blood cell count and serum potassium level in nonROSC and sustained ROSC groups. (Gray box: 1st quartile - 3rd quartile, Thick line: median, Lower end line: 1st quartile-1.5 median, Upper end line: 3rd quartile+1.5 median, point: outlier).

excluded patients, 33 had incomplete medical records, 25 had DNR orders, and blood tests were not obtained in 34 (figure 1).

\section{Study protocol}

OHCA patients were identified at triage or based on information provided by the EMS. Advance cardiac life support (ACLS) was implemented according to the 2005 America Heart Association (AHA) guidelines and included chest compression and emergent endotracheal intubation with mechanical ventilator support, intravenous injection of epinephrine, and cardioversion accordingly. (10) A blood sample was collected and sent for laboratory tests, including blood gas analysis, complete blood cell count, and biochemical studies. Sustained ROSC is defined as 20 consecutive minutes of signs of circulation without chest compression. (11) Patients with sustained ROSC are then transferred to intensive care units for further care as indicated. Survival to discharge was defined as being discharged alive or able to be transferred to a long-term care centre.

\section{Measurements}

We reviewed the charts and collected the following variables in relation to the subjects' ED visits: patient demographics (age, sex, transportation, and 
Table 1. Patient characteristics between sustained and non-sustained ROSC groups in non- traumatic OHCA patients.

\begin{tabular}{llll}
\hline Sustained ROSC & No $(\mathrm{n}=62)$ & Yes $(\mathrm{n}=60)$ & $\mathrm{p}$-value \\
\hline Gender & 46 & 35 & 0.064 \\
\hline Male & 16 & 25 & \\
\hline Female & $71.5(56-83)$ & $73.5(59-84)$ & 0.424 \\
\hline Median age in years (IQR) & & & 0.12 \\
\hline Mode of transportation & 42 & 34 & \\
\hline EMS & 17 & 12 & \\
\hline Family & 3 & 14 & 0.006 \\
\hline Other & 28 & 0.145 \\
\hline Witness collapse & 9 & 42 & 0.022 \\
\hline Bystander CPR & & 15 & \\
\hline Cardiac rhythm at ED & 54 & 40 & \\
\hline Asystole & 2 & 8 & 12 \\
\hline VF/ pulseless VT & 6 & & \\
\hline PEA & & & \\
\hline
\end{tabular}

CPR, cardiopulmonary resuscitation; ED, emergency department; EMS, emergency medical service; IQR, interquartile range; OHCA, out of hospital cardiac arrest; $\mathrm{PEA}$, pulseless electrical activity; ROSC, return of spontaneous circulation; VF, ventricular fibrillation; VT, ventricular tachycardia.

Categorized variables are given as number of patients.

Variable test using Chi-square test (Gender, Mode of transportation, Witness collapse, Bystander CPR, Cardiac rhythm), and Two sample t-test (Age)

Table 2. Findings and timing of resuscitation interventions between sustained and non-sustained ROSC groups in nontraumatic OHCA patients after arriving at the ED.

\begin{tabular}{|c|c|c|c|}
\hline Sustained ROSC & No $(n=62)$ & Yes $(n=60)$ & p-value \\
\hline Mean initial Body temperature, (SD) & $35.2(1.92)$ & $35.4(1.15)$ & 0.420 \\
\hline \multicolumn{4}{|l|}{ Timing of resuscitation interventions } \\
\hline Median time to successful intubation, minutes (IQR) & $2(1-5)$ & $2(1-3)$ & 0.524 \\
\hline $\begin{array}{l}\text { Median time to intravenous adrenaline injection, min- } \\
\text { utes (IQR) }\end{array}$ & $2(1-5)$ & $2(1-3)$ & 0.108 \\
\hline Median time to Blood drawing, minutes (IQR) & $10(6-12)$ & $7(2-14)$ & 0.838 \\
\hline \multicolumn{4}{|l|}{ Laboratory tests } \\
\hline \multicolumn{4}{|l|}{ Blood gas, mean (SD) } \\
\hline $\mathrm{pH}$ & $6.9(0.24)$ & $7.0(0.21)$ & 0.003 \\
\hline $\mathrm{PaCO}_{2}, \mathrm{mmHg}$ & $79.1(49.03)$ & $66.3(29.26)$ & 0.338 \\
\hline Bicarbonate, mm/L & $14.5(7.60)$ & $17.8(9.69)$ & 0.090 \\
\hline Capillary blood glucose, mg/dL & $224.1(147.54)$ & $261.2(127.26)$ & 0.122 \\
\hline White blood cell (WBC), 1000/uL & $10.7(5.56)$ & $14.6(7.61)$ & 0.001 \\
\hline Hematocrit, \% & $32.0(12.32)$ & $31.9(7.78)$ & 0.977 \\
\hline Creatinine, mg/dL & $3.3(6.19)$ & $1.9(1.29)$ & 0.040 \\
\hline Sodium, mEq/L & $140.0(9.80)$ & $139.6(11.23)$ & 0.449 \\
\hline Potassium, mEq/L & $7.4(2.45)$ & $5.3(1.56)$ & $<0.001$ \\
\hline AST, U/L & $409.9(983.48)$ & $313.2(1289.25)$ & 0.068 \\
\hline Creatine Kinase-MB, ng/mL & $11.9(30.18)$ & $6.9(8.94)$ & 0.488 \\
\hline Troponin I, ng/mL & $0.8(2.18)$ & $0.4(0.98)$ & 0.116 \\
\hline
\end{tabular}

AST, aspartate aminotransferase; ED, emergency department; IQR, interquartile range; OHCA, out of hospital cardiac arrest; ROSC, return of spontaneous circulation; SD, standard deviation; WBC, white blood cell.

Variable test using Mann-Whitney U test (time to successful intubation, time to intravenous adrenaline injection, PaCO2, bicarbonate, capillary blood glucose white blood cell, creatinine, AST, creatine kinase-MB, troponin I), and two sample t-test (initial body temperature, time to blood drawing, pH, hematocrit, sodium, potassium) 
Table 3. Logistic regression analysis of factors related to sustained ROSC (ROSC 20 minutes) in non-traumatic OHCA patients.

\begin{tabular}{llll}
\hline & Odds ratio & 95\% Confidence Interval & P-value \\
\hline Cardiac rhythm in the ED & & & \\
\hline Pulseless VTNF & 2.472 & $0.379-16.131$ & $0.344^{\mathrm{a}}$ \\
\hline PEA & 1.849 & $0.518-6.602$ & $0.344^{\mathrm{b}}$ \\
\hline Witness of collapse & 2.294 & $0.922-5.705$ & 0.074 \\
\hline pH determined by blood gas analysis & 1.446 & $0.158-13.276$ & 0.744 \\
\hline Leukocytosis* & 3.655 & $1.422-9.395$ & 0.007 \\
\hline Creatinine & 0.943 & $0.776-1.145$ & 0.552 \\
\hline Hyperkalemia* & 0.169 & $0.057-0.500$ & 0.001 \\
\hline
\end{tabular}

ED, emergency department; OHCA, out of hospital cardiac arrest; PEA, pulseless electrical activity; ROSC, return of spontaneous circulation; VF, ventricular fibrillation; VT, ventricular tachycardia.

a The comparison between asystole and VFNT

b The comparison between asystole and PEA

*Leukocytosis : WBC count > 11000/uL, Hyperkalemia: serum potassium level > 5.5

$\mathrm{mEq} / \mathrm{L}$

underlying disease), witness of collapse, bystander CPR, mode of transportation to the hospital (sent by family, start EMS with ambulance, or others), initial cardiac rhythm at the ED, initial body temperature, time to successful intubation (minutes), time to intravenous epinephrine injection (minutes), time to blood drawing for laboratory tests (minutes), laboratory test results including capillary blood glucose, pH, bicarbonate, $\mathrm{PaCO}_{2}$ determined by blood gas analysis, white blood cell count, hematocrit, serum sodium, serum potassium, aspartate aminotransferase (AST), creatinine, serum creatine kinase-MB, and serum troponin-I.

\section{Statistical Tests}

The data were analysed using SPSS 13.0 for Windows (SPSS, Chicago, $\mathrm{IL})$. Factors associated with sustained ROSC were analysed using the Pearson Chi-square test. The two-tailed t-test or Mann-Whitney U-test was used for continuous variables as indicated. Multivariate binary logistic regression analysis was used to identify independent factors associated with sustained ROSC. In all analyses, $\mathrm{P}<0.05$ was taken to indicate statistical significance.

\section{Results}

A total of 122 adult, non-traumatic, OHCA patients were enrolled in this study. Table 1 demonstrates patient characteristics of the sustained and non-sustained ROSC group. The median age of the patients was 73 years (interquartile range, IQR: 56 - 83 years), and the cohort included predominantly men (81 patients, $66.4 \%$ ). Witness of collapse occurred in 70 (57.4\%) cases, with 24 (19.6\%) patients receiving bystander CPR. EMS was started in 76 (62.3\%) cases. An automatic external defibrillator (AED) was applied in 50 cases, with electrical defibrillation in only one case. The most common initial rhythm seen at the ED was asystole (77.0\%), followed by pulseless electrical activity (PEA) (14.8\%) and pulseless ventricular tachycardia (VT) or ventricular fibrillation (VF) (8.2\%). The underlying diseases among the enrolled patients were as follows: stroke, $28.9 \%$; diabetes mellitus, $28.7 \%$; lung disease, $23 \%$; heart disease, $22.1 \%$; chronic kidney disease, 16.3\%; and liver disease, 7.3\%. A witnessed collapse $(P=0.006)$ and pulseless VT, VF, and PEA in the ED $(P=0.022)$ were significantly different between the two groups. More patients had received bystander CPR in the sustained ROSC group, but the difference was not statistically significant $(P=0.145)$.

The findings and timing of resuscitation interventions between the two groups after arriving at the ED are shown in table 2. There were no significant differences in initial body temperature at triage $(P=0.42)$, timing of successful intubation ( $P=0.524$ ), timing of the first intravenous epinephrine injection $(P=0.108)$, hematocrit $(P=0.977)$, and serum sodium level $(P=0.429)$ between the groups. Initial blood tests during resuscitation revealed a significant correlation between white blood cell count (WBC) count $(P=0.001)$, serum potassium level $(P<0.001)$, serum creatinine level $(P=0.04)$, $\mathrm{pH}$ value by blood gas analysis $(P=0.003)$, and sustained ROSC. More patients with leukocytosis (WBC count $>11000 / \mathrm{uL}$ ) were noted in the sustained ROSC group with a statistically significant difference $(P=0.047)$. In contrast, patients with hyperkalemia (serum potassium level > $5.5 \mathrm{mEq} / \mathrm{L}$ ) had a reduced likelihood of achieving sustained ROSC $(P<0.001)$.

Higher blood sugar (mean \pm SD: $224.1 \pm 147.54$ vs. $261.2 \pm 127.26$ $\mathrm{mg} / \mathrm{dL}, \mathrm{P}=0.122$ ) and lower cardiac enzyme levels (CK-MB, mean \pm SD: $11.9 \pm 30.18$ vs. $6.9 \pm 8.94 \mathrm{ng} /$ $\mathrm{mL}, \mathrm{P}=0.488$; troponin I, mean \pm SD: $0.8 \pm 2.18$ vs. $0.4 \pm 0.98 \mathrm{ng} / \mathrm{mL}$, $\mathrm{P}=0.116$ ) were associated with sustained ROSC, but the relationships were not statistically significant.

Multiple variable logistic regression was used to analyse the factors related to sustained ROSC, including witness of collapse, cardiac rhythm at ED, $\mathrm{pH}$ determined by blood gas analysis, leukocytosis, hyperkalemia, and cre- 
atinine levels (table 3). After logistic regression analysis adjusting for the variables mentioned above, the correlations of sustained ROSC and leukocytosis or hyperkalemia remained high (leukocytosis, $P=0.007$, odds ratio [OR] 3.655, 95\% confidence interval [Cl] 1.422-9.395; hyperkalemia, $\mathrm{P}=$ 0.001 , OR 0.169, 95\% Cl 0.057-0.500) (figure 2).

\section{Discussion}

In our study, victims of non-traumatic OHCA had received resuscitation adherent to guidelines equally after arriving at the ED. Airway management, chest compressions and establishment of peripheral venous access, with an epinephrine bolus, were done efficiently and without significant delay between the groups. There were several factors associated with sustained ROSC. First of all, although the precise time of onset of OHCA was mostly unavailable from the records, witness of collapse may indicate early resuscitation and was associated with sustained ROSC. We failed to demonstrate the effects of bystander CPR between the groups which may be due to the relatively low percentage of bystander CPR in our study population. Previous studies indicated that the percentage of bystander CPR in non-traumatic OHCA is around $32.5-36 \%$. $(1,12)$

Second, despite the small proportion of PEA and pulseless VT/VF in nontraumatic OHCA on arrival at the ED, we found a correlation with sustained ROSC.

$\mathrm{Hu}$ et al. reported that only $4.1 \%$ of victims presented with pulseless VT and VF after arriving at hospital. (13) Ko et al. reported pulseless VT and VF as the initial rhythm in $11.8 \%$ of $\mathrm{OHCA}$ in Taipei, Taiwan. (14) The prevalence of coronary heart disease is lower in Taiwan in comparison with other studies. $(15,16)$

Third, there was a high prevalence of hyperkalemia in our study population. Those patients without hyperkalemia were more likely to achieve sustained ROSC. Potassium plays an important role in cardiac electri- cal and contractile activity. Previous studies have shown that an increase in serum potassium occurs during cardiac arrest and prolonged CPR both in humans and animal models. (17-19) Several factors contribute to hyperkalemia in OHCA: 1) loss of intracellular high-energy phosphates, which occurs rapidly after the onset of ischemia, resulting in dysfunctional transcellular ionic exchange mechanisms; (20) 2) global hypoperfusion after cardiac arrest induces acidosis, which leads to potassium movement into the extracellular fluid; and 3) increased tissue breakdown by chest compression and electrical defibrillation could result in the release of potassium into the extracellular fluid. (19) Despite advances in cardiac life support, a vicious circle of hyperkalemia and cardiac arrest results in resuscitation failure. Current treatment of hyperkalemia includes calcium and sodium bicarbonate infusion, $\beta_{2}$-adrenergic agonist inhalation, insulin plus glucose water injection, and haemodialysis. Previous investigations failed to demonstrate a beneficial effect on resuscitation outcome of elemental calcium administration or sodium bicarbonate therapy during resuscitation. (21-24) However, there have been several reports of successful resuscitation and survival of hyperkalemia cardiac arrest in patients undergoing haemodialysis during cardiopulmonary resuscitation. $(25,26)$ Although hyperkalemia may be the result of prolonged tissue ischemia, we propose reconsidering elemental calcium administration and sodium bicarbonate therapy during resuscitation. A further prospective randomised control study should be conducted to verify this proposal. Serum potassium level on arrival at the ED predicts prognosis and may be useful as a parameter to monitor the efficiency of resuscitative interventions.

Fourth, leukocytosis is a marker of inflammation and is commonly seen in infection or stress, such as myocardial infarction. (27-29) In our study, leukocytosis was significantly associated with sustained ROSC. None of the previous reports addressed the relationship between WBC count and ROSC in OHCA. The present finding suggests that patients who were able to respond to acute stress with an inflammatory reaction may respond to resuscitation.

In contrast, we found no significant relationship between the severity of acidosis and sustained ROSC after adjusting for the variables mentioned above by binary logistic regression analysis. Combined respiratory and metabolic acidosis develops during cardiopulmonary arrest. Hypoxiainduced anaerobic metabolism generates lactic acid, and ventilatory failure causes carbon dioxide retention. The severity of acidosis worsens as the arrested state continues and may indicate the period of ischemia. (30) The severity of acidosis was reciprocally influenced by many factors, which may not be independent factors of sustained ROSC in OHCA.

According to the results of our study, the factors associated with sustained ROSC in adult OHCA victims were mainly determined by the patient's status of severity of illness. Initial serum potassium level and WBC count, after arriving at the ED, could serve as parameters of efficiency of prehospital resuscitation and predict the prognosis. The therapeutic implications of these observations remain to be determined. Further studies should focus on public awareness, bystander CPR and how to shorten the duration of transportation.

Limitations

The present study should be interpreted in the context of the following limitations. First, our study was retrospective, and the data were collected from a computer database and chart review. Although we made every effort to remain objective, possible errors may have been introduced. Second, this study was conducted in a university-affiliated teaching hospital, which may limit the general applicability of our findings. A comparative study with other systems would be of interest. Third, there was sampling bias due 
to the large proportion of excluded patients who received no resuscitation due to DNR orders, failure to obtain blood test results, or incomplete documentation. Nevertheless, the characteristics of the patients enrolled and the results of resuscitation were similar to those in previous studies.

\section{Conclusions}

Non-traumatic OHCA patients were appropriately resuscitated, without significant delay, after arriving at the ED. Factors associated with sustained ROSC in adult OHCA were determined by the patient's status after arriving at the ED, in particular their white blood cell count and potassium level.

\section{REFERENCES}

1. Herlitz J, Engdahl J, Svensson L, Angquist KA, Young M, Holmberg S. Factors associated with an increased chance of survival among patients suffering from an out-of-hospital cardiac arrest in a national perspective in Sweden. Am Heart J 2005;149:61-6.

2. Becker LB, Ostrander MP, Barrett J, Kondos GT. Outcome of CPR in a large metropolitan area: where are the survivors? Ann Emerg Med (1991) 20: pp 355

3. Holmberg M, Holmberg S, Herlitz J, Gårdelöv B. Survival after cardiac arrest outside hospital in Sweden. Resuscitation 1998;36:29-36

4. Soo LH, Gray D, Young T, Huff N, Skene A, Hampton JR. Resuscitation from out of- hospital cardiac arrest: is survival dependent on who is available at the scene? Heart 1999;81:47-52.

5. Bunch TJ, West CP, Packer DL, Panutich MS, White RD. Admission predictors of in-hospital mortality and subsequent long-term outcome in survivors of ventricular fibrillation out-of-hospital cardiac arrest: a population-based study. Cardiology 2004;102:41-7.

6. Jacobs IG, Finn JC, Oxer HF, Jelinek GA. CPR before defibrillation in out-of-hospital cardiac arrest: a randomized trial. Emerg Med Australas 2005;17:39-45

7. Bunch TJ, White RD, Friedman PA, Kottke TE, Wu LA, Packer DL. Trends in treated ventricular fibrillation out-of-hospital cardiac arrest: a 17-year population-based study. Heart Rhythm 2004;1:255-9.

8. Weaver WD, Cobb LA, Hallstrom AP, Fahrenbruch C, Copass MK, Ray R. Factors influencing survival after out-of-hospital cardiac arrest. J Am Coll Cardiol 1986;7:752-7.

9. Herlitz J, Eek M, Engdahl J, Holmberg M, Holmberg S. Factors at resuscitation and outcome among patients suffering from out-of-hospital cardiac arrest in relation to age. Resuscitation 2003;58:309-17.

10. ECC Committee, Subcommittees and Task Forces of the American Heart Association. 2005 American Heart Association Guidelines for Cardiopulmonary Resuscitation and Emergency Cardiovascular Care. Circulation 2005; 112:IV1.

11. Jacobs I, Nadkarni V, Bahr J, Berg RA, Billi JE, Bossaert L, et al. Cardiac arrest and cardiopulmonary resuscitation outcome reports: update and simplification of the Utstein templates for resuscitation registries. A statement for healthcare professionals from a task force of the International Liaison Committee on Resuscitation (American Heart Association, European Resuscitation Council, Australian Resuscitation Council, New Zealand Resuscitation Council, Heart and Stroke Foundation of Canada, Inter American Heart Foundation, Resuscitation Council of Southern Africa). Resuscitation 63 (2004), pp. 233-249

12. Rea TD, Cook AJ, Stiell IG, Powell J, Bigham B, Callaway CW, et al. Predicting survival after out-of-hospital cardiac arrest: role of the Utstein data elements. Ann Emerg Med 2010; 55(3):249-57.

13. Hu SC, Tsai J, Lu YL, Lan CF. EMS characteristics in an asian metropolis. Am J Emerg Med 1996;14:82-85.

14. Ko PC, Ma MH, Yen ZS, Shih CL, Chen WJ, Lin FY. Impact of community-wide deployment of biphasic waveform automated external defibrillators on out-of-hospital cardiac arrest in Taipei. Resuscitation 2004;63:167-174.

15.Chien KL, Hsu HC, Sung FC, Su TC, Chen MF, Lee YT. Hyperuricemia as a risk factor on cardiovascular events in Taiwan: the Chin-Shan community cardiovascular cohort study. Atherosclerosis 2005;183:147-155.

16. Huang CH, Ma HM, Chen WJ. Out-of-Hospital Cardiac Arrest in Taipei, Taiwan. Acta Cardiol Sin 2006;22:53-7.

17. Martin GB, Carden DL, Nowak RM, Foreback C, Tomlanovich MC. Hyperkalemia during cardiac arrest and resuscitation in the canine model. Crit Care Med 1986;14:300-302.

18. Martin GB, Nowak RM, Cisek JE, Carden DL, Tomlanovich MC. Hyperkalemia during human cardiopulmonary resuscitation: Incidence and ramifications. J Emerg Med 1989;7:109-113.

19. Niemann JT, Cairns CB. Hyperkalemia and Ionized Hypocalcemia During Cardiac Arrest and Resuscitation: Possible Culprits for Postcountershock Arrhythmias? Annals Emerg Med 1999;34(1): 1-7.

20. Jennings RB, Murry CE, Steenbergen C, Reimer KA. Development of cell injury in sustained acute ischemia. Circulation 1990;82(Suppl II): II-2-II-12.

21. Steuven H, Thompson BM, Aprahamian C, Darin JC. Use of calcium in prehospital cardiac arrest. Ann Emerg Med 1983;12:136-139. 
22. Thompson BM, Steuven HS, Tonsfeldt DJ, Aprahamian C, Troiano PF, Kastenson GH, et al. Calcium: Limited indications, some danger. Circulation 1986;74(Suppl IV):IV-90-IV-93.

23. Vukmir RB, Katz L; Sodium Bicarbonate Study Group. Sodium bicarbonate improves outcome in prolonged prehospital cardiac arrest. Am J Emerg Med 2006; 24:156.

24. Dybvik T, Strand T, Steen P. Buffer therapy during out-of-hospital cardiopulmonary resuscitation. Resuscitation 1995; $29: 89$.

25. Lin JL, Lim PS, Leu ML, Huang CC. Outcomes of severe hyperkalemia in cardiopulmonary resuscitation with concomitant hemodialysis. Intensive Care Medicine1994; 20(4):287-90

26. Kao KC, Huang CC, Tsai YH, Lin MC, Tsao TC. Hyperkalemic cardiac arrest successfully reversed by hemodialysis during cardiopulmonary resuscitation: case report. Chang Gung Medical Journal, 2000; 23(9):555-9.

27. Friedman GD, Klatsky AL, Siegelaub AB. Leukocyte count as a predictor of myocardial infarction. N Engl J Med 1974;290:1275-8.

28. Haines AP, Howarth D, North WR, Goldenberg E, Stirling Y, Meade TW, et al. Haemostatic variables and the outcome of myocardial infarction. Thromb Haemost 1983;50:800-3.

29. Furman MI, Becker RC, Yarzebski J, Savegeau J, Gore JM, Goldberg RJ. Effect of elevated leukocyte count on in-hospital mortality following acute myocardial infarction. Am J Cardiol 1996;78:945- 8.

30. Ushay HM, Notterman DA. Pharmacology of pediatric resuscitation. Pediatr Clin North Am 1997; 44:207. 